\title{
A WELL-CONNECTED WORLD
}

\section{The small but focused snapshot of research afforded by the Nature Index helps fine-tune analysis of global scientific collaboration, say Jonathan Adams and Tamar Loach.}

$\mathrm{R}$ esearch has entered a 'Fourth Age': the leading edge of scientific discovery is now in the realm of international collaboration networks rather than individuals, institutions or nations. The research that emerges from these collaborations garners more attention than national research and is cited more frequently by other publications.

The Nature Index is a selection of the world's leading research publications, honed to capture the very best articles across key research fields within the natural sciences. This provides us with a keenly focused picture of recent collaborations between world leading research institutions. Because of this emphasis, the graphics and analyses in this Nature supplement can highlight interactions more clearly than analyses that draw on the sometimes-disparate specialist and national journals in commercial databases.

The clarity of the Nature Index data presented provides a new and complementary tool for research and policy analysts, allowing us to determine which institutions are part of this growing global network, who they work with, and how their research contributes to innovation.

\section{GROUP-THINK}

The evolution of research networks between countries or institutions is of more than academic interest. If collaboration is linked with high impact, then research groups who are not part of the collaborative network risk being left behind, marginalized by a lack of access to the cutting edge of research in their field. This has obvious policy implications: it will be essential for emerging research economies to access these networks as they grow ${ }^{2}$.

In the early 1980s, the G7 group of large economies dominated global research. International collaboration was rare: the trans-Atlantic axis was the main focus of collaboration, yet joint papers with the United States accounted for less than $5 \%$ of the total output for France, Germany and even for the United Kingdom, which was its most frequent partner. Collaboration has broadened substantially since then, partly because of low-cost travel and high-speed Internet, and partly because both individuals and organizations recognize the benefits.

Collaboration often involves actual cost in travel and consumables, and always has a price in terms of time and in sharing an agenda and objectives. It is therefore reasonable to assume that when scientists collaborate they expect to achieve more and better than they can do on their own. Collaboration matters at national policy level, for example when it involves cost-sharing for astronomy or particle physics where vastly expensive facilities are required - no single country can readily afford these investments. But whether collaboration is voluntary or absolutely necessary, it nonetheless results in high-impact research.

The Nature Index points to ways in which this global structure is evolving. Considering the data for Europe, we can see that the French National Centre for Research (CNRS) and Max Planck Group — networks in their own right - and key UK universities such as Cambridge, Imperial College and Oxford provide a strong regional focal point (see 'Global clusters'). But we can also see that Spain and Portugal are drawn out of the European cluster because of their links to Latin America, a new regional network with its own structure led by Brazil, Chile, Mexico and Argentina (see S60).

Australia has historical links to Europe, particularly the UK, but the
Nature Index shows that its fastest collaborative growth is within the Asia-Pacific group and with China. This may be just one instance of the ways that traditional knowledge pipelines may be redirected and that raises a policy challenge for established partners. For example, the United Kingdom relied on historical ties to tap into Australian research: it now needs more active management to foster those global relationships.

\section{INS AND OUTS}

The Nature Index also highlights some interesting examples that buck the trend. For example, in China, there is a very strong national network revolving around the Chinese Academy of Sciences (CAS); China has a strengthening link with the United States, but it is not so connected with the Asia-Pacific network (S68).

There is also a paucity of international connections for Indian institutions and the country's national structure seems to separate corporate and academic collaborations. These differences may be a result of the unparalleled growth rate of the research bases in China and India. They have increased their absolute levels of collaboration, with expansion exceeding the capacity of global partners to engage. This is also true to a lesser extent for other emerging nations, and has two consequences. Researchers in these growing countries risk missing the benefits of sharing project development with more experienced teams in Europe and the United States; while researchers in the established research countries will lose insight into developments coming from research in these innovative regions.

The pattern at institutional level is also informative, likewise showing major variations. More than half of articles from UK institutions in the Nature Index have a co-author from a second country, most commonly the US, Germany and France. For the University of Cambridge, this figure exceeds $70 \%$. In contrast, the US average for international co-authorship is around one-third, but Harvard - by far the most collaborative US university - has an international co-author on around half of its output.

In China, CAS is the dominant player overall, but although it is ahead of the country's $25 \%$ average for international collaboration, it lags behind Peking and Tsinghua universities.

Corporate co-authorship is a tangible sign of technological and economic benefit from research. The top echelon of journals in the Nature Index shows where the corporate partners cluster: there is a strong interface between Europe and North America and throughout the AsiaPacific region, but a very sparse network in China. Note, however, that while industry may pick out the richest seams of knowledge, it is not obliged to reveal all of this in journal articles (S76).

This pattern of relatively frequent international collaboration for leading research institutions reinforces national disparities. Researchintensive institutions access and gain from networks that produce leading edge research. Other institutions, even in G7 countries, lack this level of access and if you're not at the table then you can't listen to the conversation. At a national level, the emergent research nations may be disadvantaged by that lack of connectivity.

Collaboration among Asian, European and US research organizations, recognized as the leaders in many fields, explains why international networks - captured here in the Nature Index - also deliver often-cited research output. A key policy question is whether these 


\section{GLOBAL CLUSTERS}

Institutions are sized by fractional count and connected to each of their collaborators (analogous to charged balls that repel each other, but also connected by springs). This sees them cluster with institutions with whom they collaborate frequently. Those in the middle are tightly connected with many institutions, whereas small clusters on the periphery have strong internal connections, and tend to be national groups. Some clusters are picked out below.

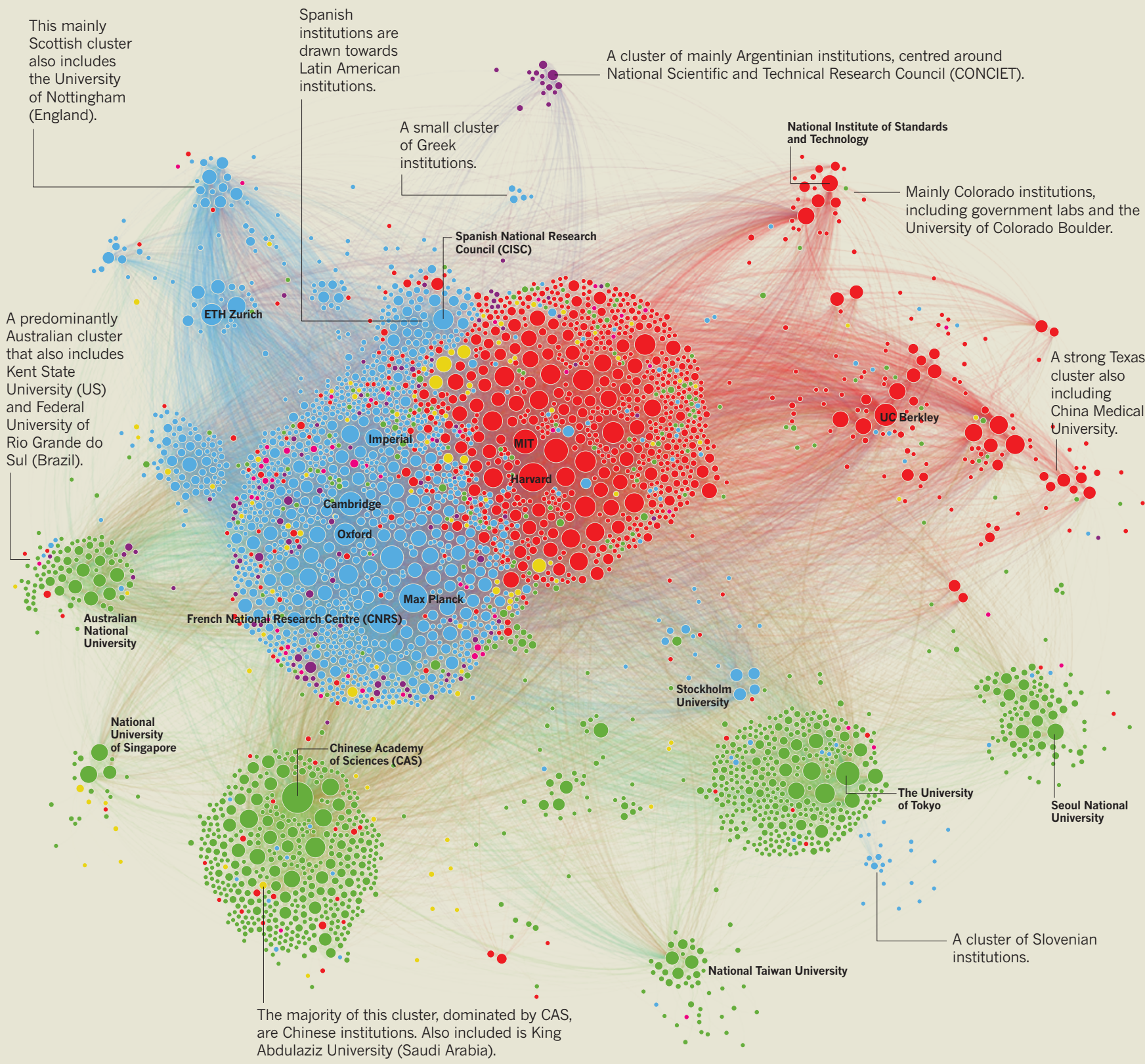

D NATURE.COM

Africa Asia Pacific North America Europe South America West Asia

To explore the graphic in more detail online, visit: go.nature.com/i2LVfl

Data shown are for 2014

For an explanation of collaboration score and other metrics, see page $\$ 83$.

national giants should also be funded to reach out to work with institutions in emerging economies that certainly need knowledge, insight and financial resources to contribute to solutions to common global challenges. Excellence talking to excellence is a powerful force, but global net benefit may come from a slightly wider discourse.
Jonathan Adams, Chief Scientist, Digital Science and visiting professor at The Policy Institute, King's College, London Tamar Loach, Research Metrics Analyst, Digital Science

1. Adams, J. Nature, 497, 557-560 (2013)

2. Leydesdorff, L. and Wagner, C.S. J. Informetr. 2, 317-325 (2008). 